\title{
Construção de curvas de energia potencial para sistemas diatômicos
}

\author{
Marcos D. S. Alves ${ }^{1} *$, Ramon S. da Silva ${ }^{1}$, Maikel Y. Ballester ${ }^{1}$ \\ ${ }^{1}$ Departamento de Física, Universidade Federal de Juiz de Fora, Juiz de Fora, MG, Brasil \\ marcosalves@ice.ufjf.br
}

\begin{abstract}
Resumo
No estudo de sistemas moleculares poliatômicos dentro do formalismo da expansão de muitos corpos, a superfície de energia potencial é construida somando os termos de interação diatômicos, triatômicos e assim por diante. Neste sentido, o objetivo do presente trabalho foi construir, utilizando-se o método da expansão dupla de muitos corpos (DMBE), curvas de energia potencial (CEP's) que representassem a topologia das diatômicas: $\mathrm{O}_{2}\left(\mathrm{X}^{3} \Sigma_{\mathrm{g}}^{-}\right), \mathrm{SO}\left(\mathrm{X}^{3} \Sigma^{-}\right)$e $\mathrm{N}_{2}\left(\mathrm{X}^{1} \Sigma_{\mathrm{g}}^{+}\right)$em seus correspondentes estados eletrônicos fundamentais.
\end{abstract}

\section{Introdução}

No estudo de moléculas diatômicas é possível separar a equação de Schrodinger em uma parte eletrônica e outra nuclear valendo-se da aproximação BornOppenheimer. Neste sentido, a interação entre os núcleos é dada por uma curva de energia potencial. Por sua vez, esta desempenha um papel importante na análise de estudos envolvendo estrutura [1] e dinâmica molecular [2]. A CEP pode ser descrita por uma função analítica ajustada de todas as posições relativas dos átomos e/ou moléculas a partir de um conjunto de pontos $a b$ initio e/ou experimentais. Após a construção de uma CEP acurada é possível obter informação do sistema diatômico, como por exemplo predizer o espectro ro-vibracional e o processo de dissociação.

Funções como a de Morse e o método
DMBE [3] são exemplos de formas funcionais que simulam bem a topologia de uma CEP. Neste trabalho, é dado foco principal ao método DMBE no ajuste dos pontos obtidos com o auxílio do MOLPRO, um pacote para cálculos $a b$ initio [4].

\section{Metodologia}

No Molpro foram gerados 500 pontos $a b$ initio para cada sistema diatômico de interesse, bem como a otimização de geometria. Exceto para o $\mathrm{O}_{2}$, que teve pontos gerados com nível de cálculo CASPT2, nas demais diatômicas utilizou-se o nível MRCI(Q). O conjunto de bases de Dunning $\operatorname{AVXZ}(\mathrm{X}=4,5)$ foram adotadas neste trabalho. Tendo em mãos os pontos $a b$ initio, foi possível ajustá-los valendo-se do método DMBE. De acordo com este 


\section{Blucher Proceedings \\ VIII Encontro Científico de Física Aplicada Blucher}

método, a CEP é dividida em duas partes: uma que contabiliza a energia de longo alcance ou dynamical correlation (dc) e a outra descrevendo o curto alcance ou energias extended Hartree-Fock (EHF). Portanto temos:

$$
V(R)=V_{E H F}(R)+V_{d c}(R)
$$

$V(R)$ deve satisfazer os limites assintóticos $\mathrm{R} \rightarrow 0$ e $\mathrm{R} \rightarrow \infty$. Uma das formas utilizadas para avaliar a qualidade das CEP's ajustadas foi calcular o erro quadrático médio $\left(\Delta E_{R M S D}\right)$ dado por:

$$
\Delta E_{R M S D}=\frac{1}{N}\left[\sum_{i=1}^{N}\left(V_{\text {fit }}-V_{\text {abinitio }}\right)\right]^{1 / 2}
$$

\section{Resultados}

\section{$3.1 \mathrm{O}_{2}\left(\mathrm{X}^{3} \Sigma_{\mathrm{g}}^{-}\right)$}

Na Fig. 1] é mostrado a CEP para o sistema diatômico $\mathrm{O}_{2}$ em seu estado eletrônico fundamental e erro associado, o qual apresenta diferença entre o ajuste e os pontos $a b$ initio menores que 0,04 $\mathrm{kcal} / \mathrm{mol}$. Após a otimização da geometria, obteve-se o valor de 1,2121 $(1,2075) \AA ̊$ e $-0,1902 \mathrm{E}_{\mathrm{h}}$ para a distância de equilíbrio $\left(\mathrm{R}_{\mathrm{e}}\right)$ e energia, respectivamente. Os valores calculados para a constante rotacional na posição de equilíbrio $\left(B_{e}\right)$ e frequência de vibração $\left(\omega_{\mathrm{e}}\right)$ foram de $1,4317(1,4376)$ e $1565,16(1580,19)$, ambos $\mathrm{em} \mathrm{cm}^{-1}$. Estes valores quando comparados aos encontrados no NIST ${ }^{1}$ mostram bom acordo com erro relativo associado de $3 \%$.

\footnotetext{
${ }^{1}$ Valores do NIST estão em parênteses.
}
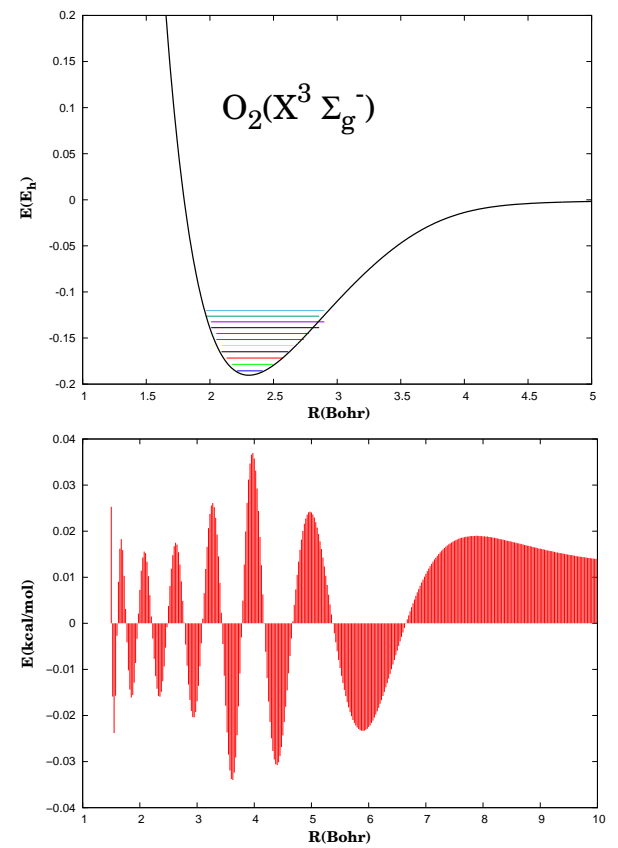

Figura 1: CEP para a diatômica $\mathrm{O}_{2}\left(\mathrm{X}^{3} \Sigma_{\mathrm{g}}^{-}\right)$ e seu erro associado. As linhas coloridas no painel superior indicam níveis vibracionais de energia onde variou-se o número quântico $\mathrm{v}$ de 0 a 10 .

\section{2 $\mathrm{SO}\left(\mathrm{X}^{3} \Sigma^{-}\right)$}
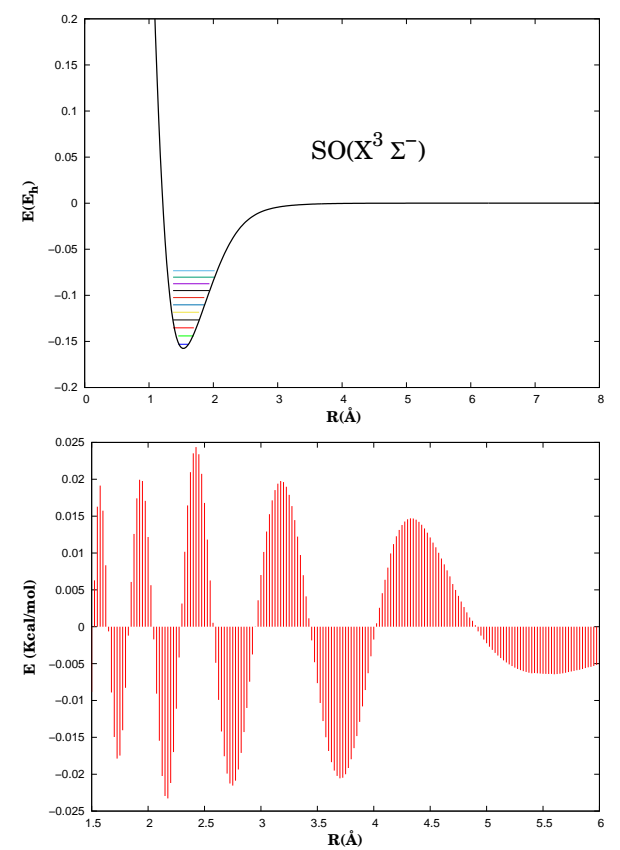

Figura 2: CEP para a diatômica $\mathrm{SO}\left(\mathrm{X}^{3} \Sigma^{-}\right) \mathrm{e}$ seu erro associado. 


\section{Blucher Proceedings VIII Encontro Científico de Física Aplicada Blucher}

Para a molécula de monóxido de enxofre foi repetido o mesmo procedimento descrito anteriormente, entretanto, encontrou-se para os pontos ajustados e aqueles $a b$ initio diferenças menores que 0,025 kcal/mol (Ver Fig. 2). As linhas coloridas no painel superior indicam níveis vibracionais de energia onde variou-se o número quântico v de 0 a 10 . Os resultados obtidos após otimizar a geometria são $\mathrm{R}_{\mathrm{e}}=1,5061(1,4810) \AA$ com energia associada de $-0,1642 \mathrm{E}_{\mathrm{h}}$. Obteve-se para $\mathrm{B}_{\mathrm{e}}$ e $\omega_{\mathrm{e}}$ os valores de 0.6937 (0.7208) $\mathrm{cm}^{-1}$ e $1078,67(1149,20) \mathrm{cm}^{-1}$, respectivamente. Todos os valores encontrados para o SO também mostram concordância com aqueles do NIST tendo erro relativo associado de $3 \%$, menos o valor de frequência cuja diferença é aproximadamente $7 \%$.

\section{3 $\mathrm{N}_{2}\left(\mathrm{X}^{1} \Sigma_{\mathrm{g}}^{+}\right)$}
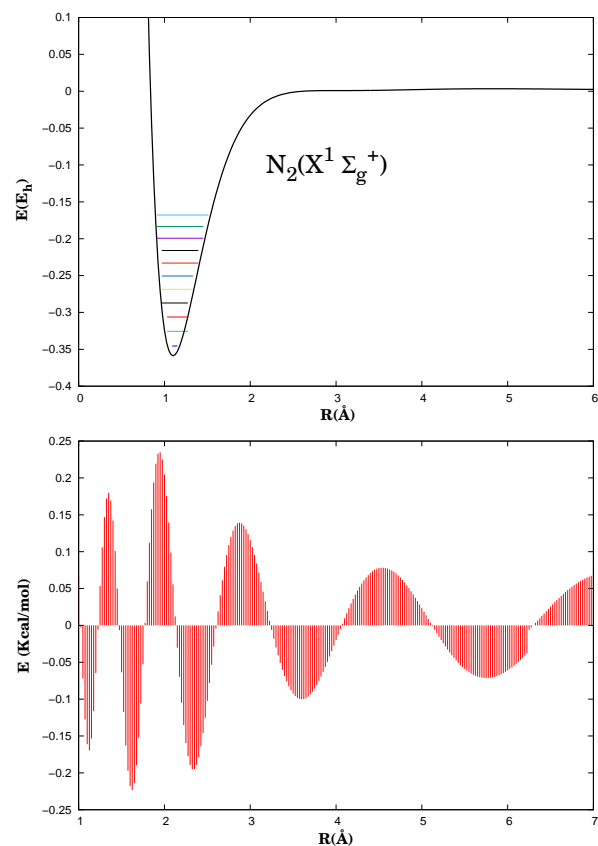

Figura 3: CEP para a diatômica $\mathrm{N}_{2}$ $\mathrm{N}_{2}\left(\mathrm{X}^{1} \Sigma_{\mathrm{g}}^{+}\right)$e seu erro associado.

Para o $\mathrm{N}_{2}$, as diferenças entre os pontos ajustados e aqueles $a b$ initio são menores que $0,25 \mathrm{kcal} / \mathrm{mol}$ (Ver Fig. 3).
Os resultados obtidos após otimizar a geometria foram de $\mathrm{R}_{\mathrm{e}}=1,0950(1,0976)$ $\AA ̊$ com energia associada de $-0,3672 \mathrm{E}_{\mathrm{h}}$. Calculou-se para $\omega_{\mathrm{e}} \mathrm{o}$ valor de 2411,31 $(2358,57) \mathrm{cm}^{-1}$ com constante rotacional $\mathrm{B}_{\mathrm{e}}=1,9982(1,9982) \mathrm{cm}^{-1}$. O erro relativo associado para o $\mathrm{N}_{2}$ mostrou-se ser de $3 \%$.

\section{Conclusão}

A partir do ajuste dos pontos $a b$ initio construiu-se CEP's para os sistemas diatômicos de interesse. A qualidade dos ajustes pôde ser determinada por um conjunto de informações, como cálculo de $\mathrm{R}_{\mathrm{e}}, \omega_{\mathrm{e}}$ e $\mathrm{B}_{\mathrm{e}}$. O valor de $\Delta E_{R M S D}$ encontrado para todos os ajustes realizados na ordem de $10^{-4} \mathrm{E}_{h}$ foi considerado como um indício da boa qualidade. A obtenção de funções analíticas razoavelmente precisas para uma CEP diatômica contribuirá na descrição de sistemas contendo um maior número de átomos.

\section{Agradecimentos}

Os autores agradecem a CAPES, FAPEMIG e BIC/UFJF pelo apoio financeiro.

\section{Referências}

[1] GARRIDO, J. D. et al. J. Phys. Chem. A, v. 115, p. 1453-1461, 2011.

[2] PIRES, W. A. D. et al. Phys. Chem. Chem. Phys., v. 16, p. 12793-12801, 2014.

[3] VARANDAS, A. J. C. Adv. Chem. Phys, v. 74, p. 255-338, 1988.

[4] WERNER, H. et al. MOLPRO, version 2010.1, a package of ab initio programs, 2010. Disponivel em http://www.molpro.net 